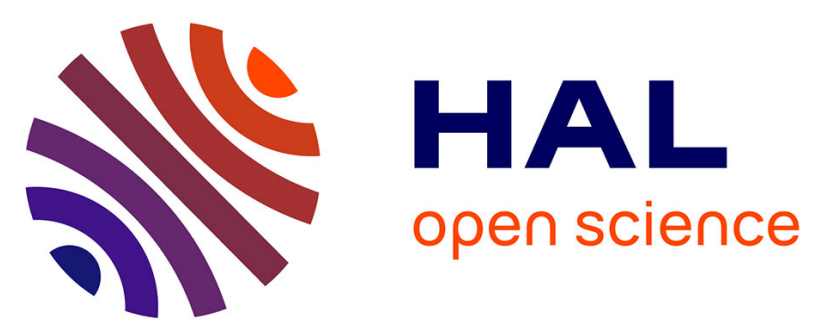

\title{
Patterning highly conducting conjugated polymer electrodes for soft and flexible microelectrochemical devices
}

\author{
Alexandre Khaldi, Daniel Falk, Katarina Bengtsson, Ali Maziz, Daniel \\ Filippini, Nathaniel D Robinson, Edwin W H Jager
}

\section{To cite this version:}

Alexandre Khaldi, Daniel Falk, Katarina Bengtsson, Ali Maziz, Daniel Filippini, et al.. Patterning highly conducting conjugated polymer electrodes for soft and flexible microelectrochemical devices. ACS Applied Materials \& Interfaces, 2018, 10.1021/acsami.8b01059 . hal-01825918

\section{HAL Id: hal-01825918 https://imt-atlantique.hal.science/hal-01825918}

Submitted on 28 Jun 2018

HAL is a multi-disciplinary open access archive for the deposit and dissemination of scientific research documents, whether they are published or not. The documents may come from teaching and research institutions in France or abroad, or from public or private research centers.
L'archive ouverte pluridisciplinaire HAL, est destinée au dépôt et à la diffusion de documents scientifiques de niveau recherche, publiés ou non, émanant des établissements d'enseignement et de recherche français ou étrangers, des laboratoires publics ou privés. 


\title{
Patterning highly conducting conjugated polymer electrodes for soft and flexible microelectrochemical devices
}

Alexandre Khaldi†*, Daniel Falk, Katarina Bengtsson, Ali Maziz广, Daniel Filippini, Nathaniel

D. Robinson, Edwin W.H. Jager*

Linköping University, Department of Physics, Chemistry and Biology, 58183 Linköping, Sweden

KEYWORDS: Vapor phase polymerization, Printing, soft Lithography, Conjugated Polymers actuators, patterning, microfabrication

\begin{abstract}
There is a need for soft actuators in various biomedical applications in order to manipulate delicate objects such as cells and tissues. Soft actuators are able to adapt to any shape and limit the stress applied to delicate objects. Conjugated polymer actuators, especially in the so-called trilayer configuration, are interesting candidates for driving such micromanipulators. However, challenges involved in patterning the electrodes in a trilayer with individual contact have prevented further development of soft micromanipulators based on conjugated polymer actuators. To allow such patterning, two printing-based patterning techniques have been developed. First an oxidant layer is printed using either syringe-based printing or micro-contact printing, followed by vapor phase polymerization of the conjugated polymer. Sub-millimeter patterns with electronic conductivities of $800 \mathrm{~S} \cdot \mathrm{cm}^{-1}$ are obtained. Next, laser ablation is used to
\end{abstract}


cleanly cut the final device structures including the printed patterns, resulting in fingers with individually controllable digits and miniaturized hands. The methods presented in this paper will enable integration of patterned electrically active conjugated polymer layers in many types of complex 3-D structures.

\section{Introduction}

The recent and rapid development of bioelectronic devices such as implantable or wearable electronics, neural interfaces, drug delivery systems, and soft actuators have highlighted the need for soft, flexible, and stretchable electrodes and wires. Conjugated polymers (CPs) are the materials of choice for interfacing electronic devices with natural tissue and cells due to their combination of electronic and ionic conductivity, biocompatibility, low cost, the ease of processing, and their ability to undergo reversible oxidation and reduction reactions. ${ }^{1-7} \mathrm{CPs}$ with good chemical stability exhibiting electronic conductivity above $1000 \mathrm{~S} \cdot \mathrm{cm}^{-1}$ are now common. ${ }^{8-}$ ${ }^{10}$ However, these CP layers are frequently coated without patterning, and must therefore be patterned, for instance, to form individual electrodes, in order to obtain functional devices. ${ }^{11} \mathrm{~A}$ variety of techniques have been used to pattern these materials at the micro/nano scale. ${ }^{12,13}$ This can be achieved by electropolymerization onto patterned gold electrodes, but the poor adhesion of the gold layer to both the $\mathrm{CP}$ and the underlying substrate limits device lifetime. ${ }^{12-16}$ The subtractive patterning of a CP layer by laser ablation ${ }^{17}$ or reactive ion etching ${ }^{18}$ is extremely difficult to perform on multi-layered materials, as it is difficult to ensure that only the CP 
material is removed without disturbing any underlying polymer layers. Over-oxidation (electrochemical or oxidative deactivation) of CP layers can also be used for patterning, but this method only locally disrupts the electrical conductivity, while physical removal of the electrode material is also often required. ${ }^{19}$ Rational fabrication of bioelectronics devices based on CPs requires new patterning techniques that are compatible with batch fabrication processes that use bottom-up microfabrication techniques. PEDOT-PSS dispersed in water, e.g. Clevios $\mathrm{S}^{\mathrm{TM}}$ from Heraeus, is directly patternable by screen printing, ${ }^{20}$ is able to present high electronic conductivities $^{21,22}$ but has diminished electrochemical capacity per unit mass due to the mass contribution of the counterions and additives used. In the case of actuator applications, vapor phase polymerization (VPP), in combination with reactive ion etching, has enabled batch fabrication of simple $\mathrm{CP}$ actuator devices that can operate in air. ${ }^{10,18,23}$

$\mathrm{CP}$ actuators utilize the volumetric expansion caused by the oxidation or reduction of the $\mathrm{CP}$. A typical $\mathrm{CP}$ actuator structure (see Figure 1) is the so-called trilayer configuration, where the outer layers consist of CPs and the middle layer is a polymer electrolyte (ion reservoir). When a voltage is applied, the CP layer at the cathode is reduced and the CP layer at the anode is oxidized. Cations and/or anions migrate, compensating the charges formed on the CP chains, maintaining electroneutrality, and inducing opposing volumetric changes in the electrodes. The simultaneous expansion and contraction of the $\mathrm{CP}$ layers sandwiching the polymer electrolyte causes the trilayer beam to bend. This bending is reversible, so applying an alternating potential causes the beam to oscillate. ${ }^{24,25}$ 


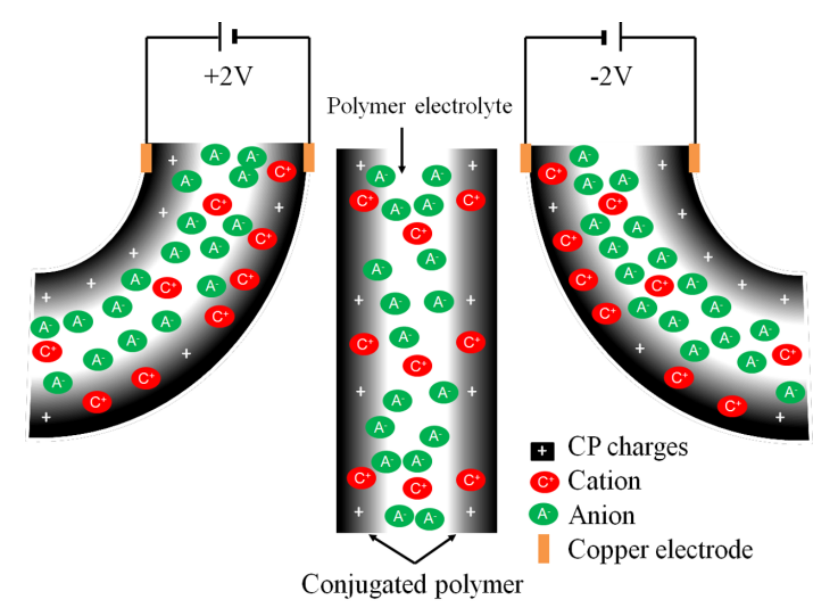

Figure 1. Schematic showing CP trilayer actuation. The dark layers represent the CP layers, which sandwich an ionic conductive polymer electrolyte membrane, shown in white. Three different actuation states are presented: the unbiased state (center), and the positively and negatively biased states (left and right, respectively). Note that the device is symmetric and that the applied potential defines the direction.

These trilayer $\mathrm{CP}$ actuators, operable in air, have been scaled down to the micro-domain, and multiple bending beam type microactuators have been fabricated in parallel. ${ }^{26}$ Unfortunately, the previously-reported parallel actuators could not be operated individually as the micropatterning method did not allow individual independent electrical connections. Individually controlled actuation has been demonstrated at the centimeter scale using classically-micropatterned metal electrodes with electrodeposited polypyrrole (PPy) ${ }^{27}$ However, the latter method required flipping the substrate during processing, making the alignment of the various layers difficult, hampering microfabrication and hindering scale-up and commercial development. To pattern CP electrodes at microscale resolution, we recently developed a bottom-up microfabrication method; unfortunately, these trilayer actuators did not show the large deformations expected with $\mathrm{CP}$ actuators because the electrolyte membranes used had relatively low ionic conductivity. ${ }^{28,29} \mathrm{~A}$ few attempts have been made to obtain micropatterned CP layers using soft lithography and 
VPP, but the adhesion of the resulting electrode was insufficient for devices undergoing large deformations. ${ }^{11,30}$

Here, we present two patterning methods, micro-Contact Printing $(\mu \mathrm{CP})$ and syringe-based printing, suitable for VPP. Although we focus on CP actuator processing in this paper, these methods are suitable for microfabricating many kinds of devices comprising CPs. These two methods allow the patterning of micro- to millimeter-sized electrodes and wires. The resolution of each patterning technique was evaluated by printing circular or square micrometer-sized shapes for $\mu \mathrm{CP}$ and millimeter-sized lines for syringe-based printing. The resolution achieved with both techniques enabled us to demonstrate active $\mathrm{CP}$ electrodes connected to contacts with thin addressing lines. Finally, the capabilities of the novel patterning methods are demonstrated by fabricating a soft actuator based on $\mathrm{CP}$ technology.

\section{Results and discussion}

\subsection{Polymer electrolyte}

We have chosen to use the semi-interpenetrating network (IPN) poly(ethylene oxide)nitrilebutadienerubber (PEO-NBR) to obtain a robust solid polymer electrolyte (SPE) with sufficient ionic conductivity. ${ }^{10}$ To fabricate high performance $\mathrm{CP}$ actuators, the center polymer electrolyte layer (Figure 1) should be a membrane swollen by an electrolyte with a high ionic conductivity, allowing efficient ion migration between the adjacent $\mathrm{CP}$ electrodes, and capable of high bending deformation without fracture. This currently limits the choice to three membrane materials: poly(ethylene oxide)-nitrilebutadienerubber interpenetrating network (PEO-NBR IPN) membrane, ${ }^{31,32}$ the commercial porous polyvinylidene fluoride (PVDF) membrane ${ }^{33}$ and the

PVDF ionic liquid gel membrane. ${ }^{34}$ The porous PVDF membrane cannot be fabricated on a 
substrate using spin coating. The PVDF ionic liquid gel is difficult to use because common patterning sequences require immersing the membrane in various solutions, leading to leaching of ionic liquids out of the membranes and limiting the robustness of such processes. Hence, the semi-IPN PEO-NBR was chosen.

\subsection{Vapor-phase polymerized PEDOT}

VPP methods have shown to be suitable for obtaining strong adhesion between CP electrodes and the membranes described above. VPP is a heterogeneous oxidative polymerization reaction between an oxidative surface and a gaseous monomer. VPP was carried out for $30 \mathrm{~min}$ at $40 \mathrm{C}$ under a vacuum atmosphere of $1 \mathrm{mPa}$ (Figure $2 \mathrm{a})$.

We have previously used VPP to fabricate CP trilayer actuators with an interpenetrating polymer network based on PEO-NBR as the center electrolyte layer. ${ }^{31,32}$ The process started with VPP of PEDOT on the substrate, followed by the PEO-NBR deposition and polymerization, and finished with a second VPP of PEDOT on top of the membrane. ${ }^{35}$ The structure was cured for 1 hour at $80^{\circ} \mathrm{C}$ to obtain crosslinking at the interfaces (described below). ${ }^{10}$

\subsubsection{Effect of PEGM-PEGDM on polymerization}

Oxidant solutions were mixed to yield between 0 and $50 \mathrm{wt} . \%$ ethylene oxide methacrylates (PEGM-PEGDM, see Experimental section) added to $40 \mathrm{wt} \% \%$ Fe (Tos) ${ }_{3}$ oxidant solution (in Ethanol/Butanol mixture). It has been shown that various additives and ethylene oxide derivatives $^{9,30,36}$ help to reduce the crystallization of $\mathrm{Fe}$ and reduce the rate of polymerization, leading to highly ordered CP films with high electronic conductivity (on the order of $10^{3} \mathrm{~S} \cdot \mathrm{cm}^{-}$ $\left.{ }^{1}\right) .{ }^{37,38}$ The acrylate oligomers in our oxidant solution promote adhesion of the CP layer to acrylic 
substrates with the addition of a post-processing step that induces the formation of covalent bonds between the layers. ${ }^{10}$ Figure $2 \mathrm{~b}$ reports the thicknesses and the conductivity of the $\mathrm{CP}$ layers resulting from VPP with solutions with varying PEGM-PEGDM content. VPP carried out with an oxidant solution without the ethylene oxide methacrylates leads to a thickness of $100 \mathrm{~nm}$. The highest measured conductivity was $800 \mathrm{~S} \cdot \mathrm{cm}^{-1}$ for a film of $200 \mathrm{~nm}$ thickness prepared from an oxidant solution containing $30 \mathrm{wt} \%$ PEGM-PEGDM. The high conductivity of this CP layer allows (metal-free) electropolymerization of subsequent polymer layers. ${ }^{35,39}$
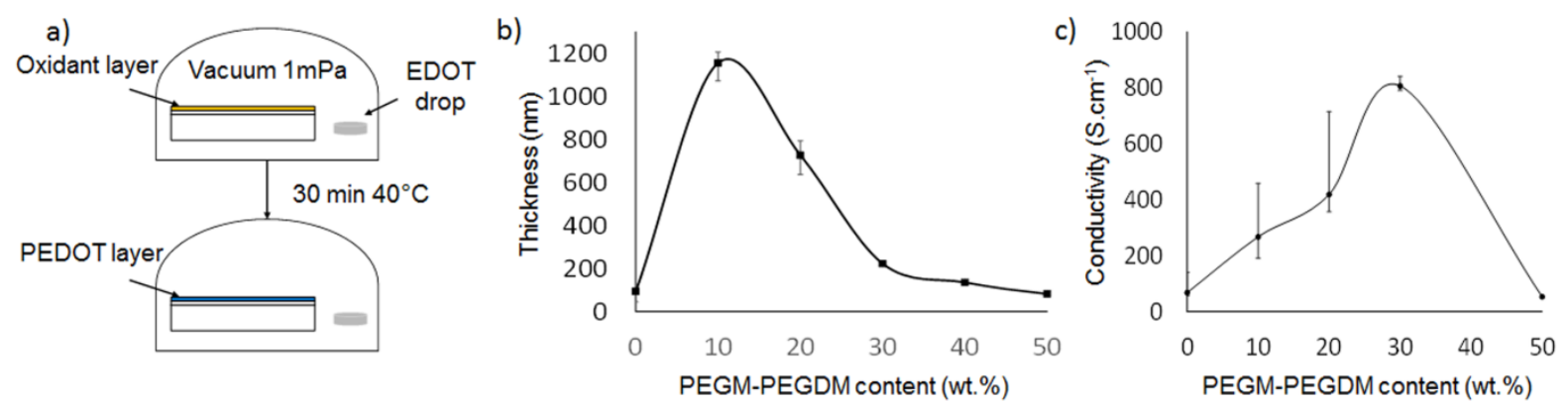

Figure 2. Vapor phase polymerization a) Schematic of the process, b) Thickness, and c) conductivity of the resulting PEDOT layer as a function of PEGM-PEGDM content used in the oxidant solution.

\subsubsection{Effect of PEGM-PEGDM on surface interaction}

Interactions between the oxidant solution and several substrates used in our patterning techniques have been analyzed. The contact angle $(\Theta)$ of the oxidant solution with varying PEGM-PEGDM content on different substrates is presented in Figure 3. We measured the contact angle of the oxidant solutions on PDMS with and without $\mathrm{O}_{2}$ plasma treatment, glass, polyvinyl alcohol (PVA), PEO-NBR IPN, and PVDF. 


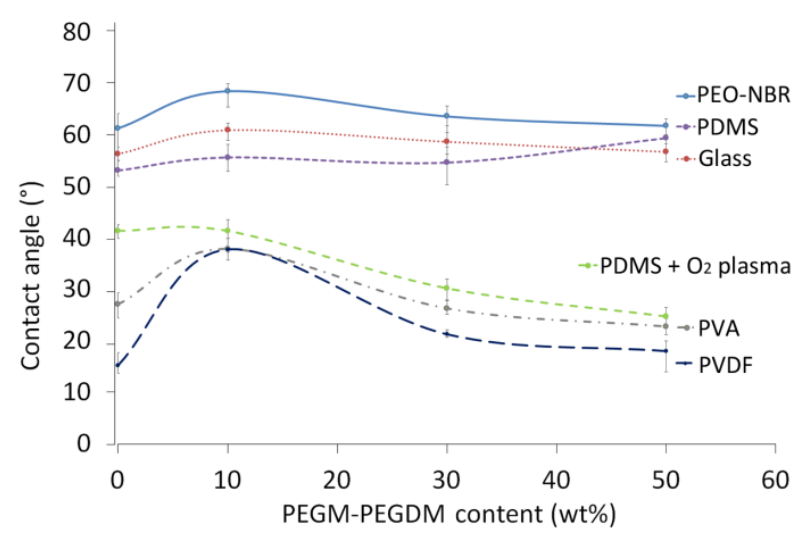

Figure 3. Contact angles of the oxidant solution on several substrates, as a function of the PEGM-PEGDM content in the solution.

The contact angle of the oxidant solution for each of the formulations was very high (above $50^{\circ}$ ) on PDMS, glass, and PEO-NBR substrates. When the PDMS was treated with an $\mathrm{O}_{2}$ plasma, the observed contact angle decreased significantly. The oxidant solution had a stronger interaction with the PVDF and PVA substrates, but the contact angle surprisingly increased to $38^{\circ}$ when we add 10 wt.\% PEGM-PEGDM to the oxidant solution. The value of the contact angle on the PVDF and PVA decreased again with increasing PEGM-PEGDM content. The tunable affinity between the oxidant solution and substrates has consequences on the quality of the reproduction of patterns after polymerization, as described in the coming sections.

\subsection{Oxidant patterning}

To achieve highly conductive high-resolution patterns of $\mathrm{CP}$ electrodes, we first pattern the oxidant solution and then perform VPP, instead of directly printing or patterning a CP layer. As mentioned previously, two different techniques have been developed to pattern the oxidant solution: $\mu \mathrm{CP}$ and syringe-based printing. For $\mu \mathrm{CP}$, square patterns were created on a PDMS 
stamp and used to verify the

technique's ability to reproduce shapes with sharp corners. The $\mu \mathrm{CP}$ process, and syringe-based printing are illustrated in Figure 4. After patterning the oxidant with one of these techniques, VPP is performed on the oxidant, forming a nicely-patterned CP layer. Next, a multi-layered structure comprising a polymer electrolyte and a second patterned CP layer can be formed, which thereafter can be processed using laser ablation (Figure 4.) 


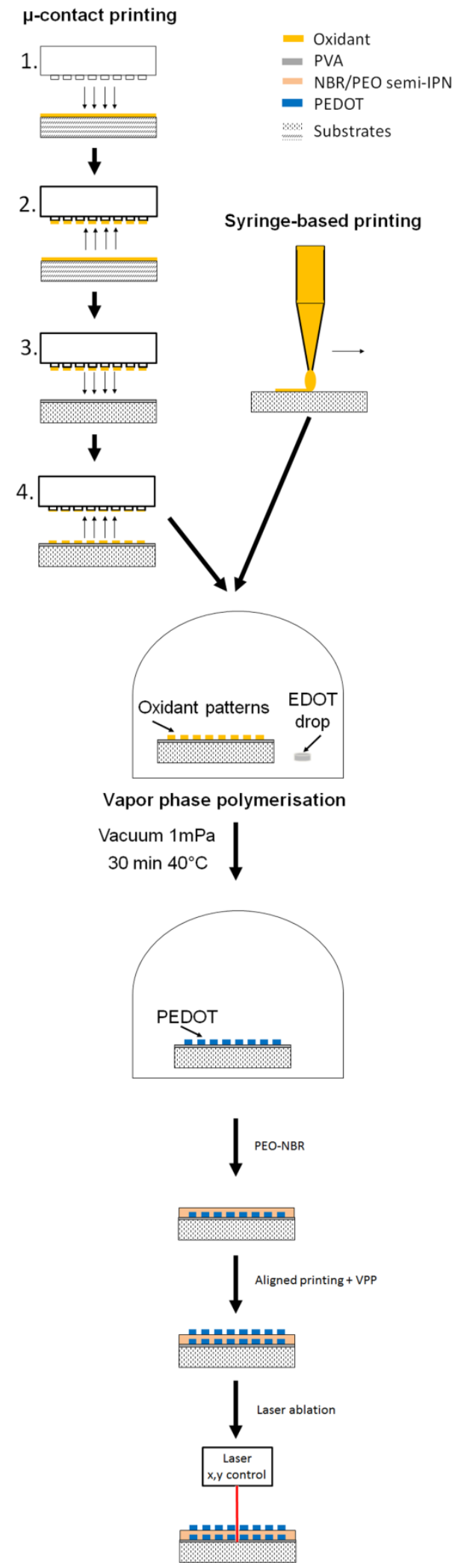


Figure 4. Process flow of the fabrication of conjugated polymer actuators: $\mu$-contact printing and syringe-based printing techniques for use with vapor phase polymerization. 1) The PDMS stamp is pressed onto the oxidant solution deposited by spin-coating on a glass slide. 2) The PDMS stamp is removed from the substrate and the oxidant solution adheres to the pattern of the PDMS stamp. 3) The oxidant pattern is reproduced onto a substrate covered by either a PVA or a PVDF layer. 4) The stamp is removed, leaving the oxidant pattern deposited onto the substrate. The syringe-based printing must be realized onto a glass slide. The vapor phase polymerization is following the creation of this oxidant pattern. A polymer electrolyte layer can be deposited before the deposition of a subsequent CP layer. The laser ablation is used to cut cleanly the final device.

\subsection{1 $\mu$-contact printing}

The interaction between the fluid (oxidant solution) and stamp and substrates are particularly important when using $\mu \mathrm{CP}$, as these interactions influence the resolution and shape of the resulting pattern. To transfer the oxidant solution from a temporary substrate (onto which the oxidant solution was applied via spin coating) to the stamp, the affinity of the oxidant solution for the temporary substrate must be less than its affinity for the stamp. Surface-treating the PDMS stamp with oxygen plasma dramatically increases the solution's affinity for the PDMS. In the second step of the process, the solution's affinity for the target substrate should be even greater than its affinity for the PDMS stamp. The contact angle measurements for all compositions of the oxidant solution show that the solutions affinity for glass is less than that for plasma-treated PDMS, which in turn is less than its affinity for targets substrate of either PVDF or PVA. In other words, the process outlined in figure 3 moves the liquid from substrates on which it has the highest contact angle to materials on which it has progressively lower contact angle $\left(\theta_{\mathrm{Glass}}>\theta_{\mathrm{PDMS}}>\theta_{\mathrm{PVA}}\right)$. The combination of $\mu \mathrm{CP}$ with vapor phase polymerization allowed us to easily create well-defined micro-patterned electrodes with high conductivity. Figures 5a-b 
show the micro-patterns obtained on a PVA substrate. It shows that it is possible to produce highly defined micro-patterns on such a surface. This technique allows reproduction of different patterns with sizes between 100 and $500 \mu \mathrm{m}$ and a minimum spacing distance of $50 \mu \mathrm{m}$ onto both PVA and PVDF substrates (Figure S1). Circular, rather than square, patterns are obtained when $10 \mathrm{wt} . \%$ PEGM-PEGDM is present in the oxidant solution, while more accurate replication of the square pattern of the stamp was obtained for $30 \mathrm{wt} . \%$ additive content in the oxidant solution onto both PVA and PVDF substrates. This observation is consistent with the maximum in measured contact angle between the oxidant solution and all substrates. This result indicates that that we can fine-tune the shape of the pattern using the formulation of the oxidant solution, which we believe warrants further study.

One advantage of $\mu \mathrm{CP}$ is that a complete layer of complex device architecture can be patterned in a single step. To demonstrate this, we made a PDMS stamp with the actuator lay-out of the first layer comprising two individually controllable electrodes/actuators, leads/wires, and contact pads. We used $\mu \mathrm{CP}$ to fabricate millimetric electrodes with $600 \mu \mathrm{m}$ wide conducting lines in a single printing step (Figure 5c). The high conductivity obtained for the electrodes allowed us to cover the thin PEDOT electrodes with a PPy layer in a subsequent electropolymerisation step with homogeneous thickness $(8 \mu \mathrm{m}$, Figure $5 \mathrm{c})$.

Unfortunately, we have been unable to use this technique to fabricate $\mathrm{CP}$ electrodes on the highly ionically conductive membrane PEO-NBR due to the insufficient affinity between the oxidant solution and the PEO-NBR membrane $\left(\Theta>60^{\circ}\right)$ compared to affinity for the plasma treated PDMS stamp $\left(\Theta<40^{\circ}\right) . \mathrm{O}_{2}$ plasma treatment of the PEO-NBR membrane reduces the surface energy (measured contact angle), but induces other problems such as penetration of the 
oxidant solution into the membrane, causing the membrane to swell. If one wants to fabricate patterned multilayered CP device with this method, new SPE membranes need to be developed.

a)

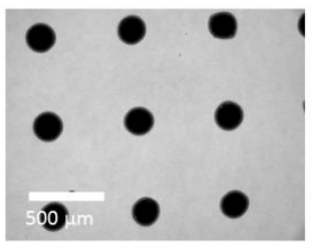

b)

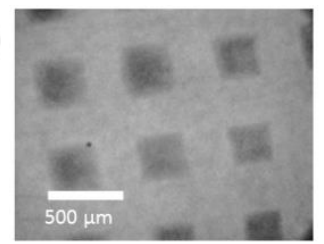

c)

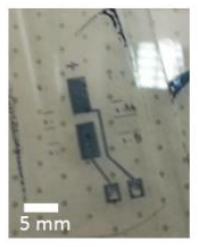

Polypyrrole

Electropolymerisation

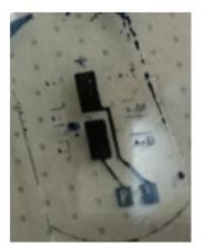

Figure 5. $\mu$ CP PEDOT patterns. a) Pattern on PVA obtained after 30 min of VPP onto a $\mu$ CP-patterned oxidant layer with $10 \%$ PEGM-PEGDM, b) The $\mu \mathrm{CP}$ pattern obtained with the same process but with $30 \%$ PEGMPEGDM, c) Using $\mu \mathrm{CP}$, large electrodes $\left(\approx 10 \mathrm{~mm}^{2}\right)$ with contact pads and connecting lines $(600 \mu \mathrm{m}$ wide $)$ were obtained with VPP on an oxidant layer with $10 \mathrm{wt} \%$ PEGM-PEGDM in a single printing step. These active electrodes can then be homogeneously covered by a thick layer of PPy using electropolymerisation. Note that the contact pads were not exposed to the electropolymerisation solution and thus are not covered with PPy.

\subsubsection{Syringe-based printing}

For syringe-based printing, a different interaction between the oxidant solution and the substrate is necessary. To prevent the solution from spreading onto the substrate, but rather retain the shape of the pattern being printed, the affinity of the solution with the substrate should be relatively low $\left(\Theta>50^{\circ}\right)$. Both glass and PEO-NBR substrates have a surface energy appropriate for patterning the oxidant layer using syringe-based printing, thus enabling the fabrication of patterned CP trilayer devices. 
Figure 6 shows the principle of fabricating individually controlled trilayer actuators with individual contacts on the same polymer film using syringe-based printing. The line resolution of the patterns obtained in this work was $\sim 1 \mathrm{~mm}$. The resolution is limited by the size of the needle, solution viscosity, the oxidant solution interaction between the tip of the needle and the substrate, the gap between needle and substrate, and the spreading of the material on the surface. Further optimization of these printing parameters would allow higher resolution patterns to be printed.

a) Top View

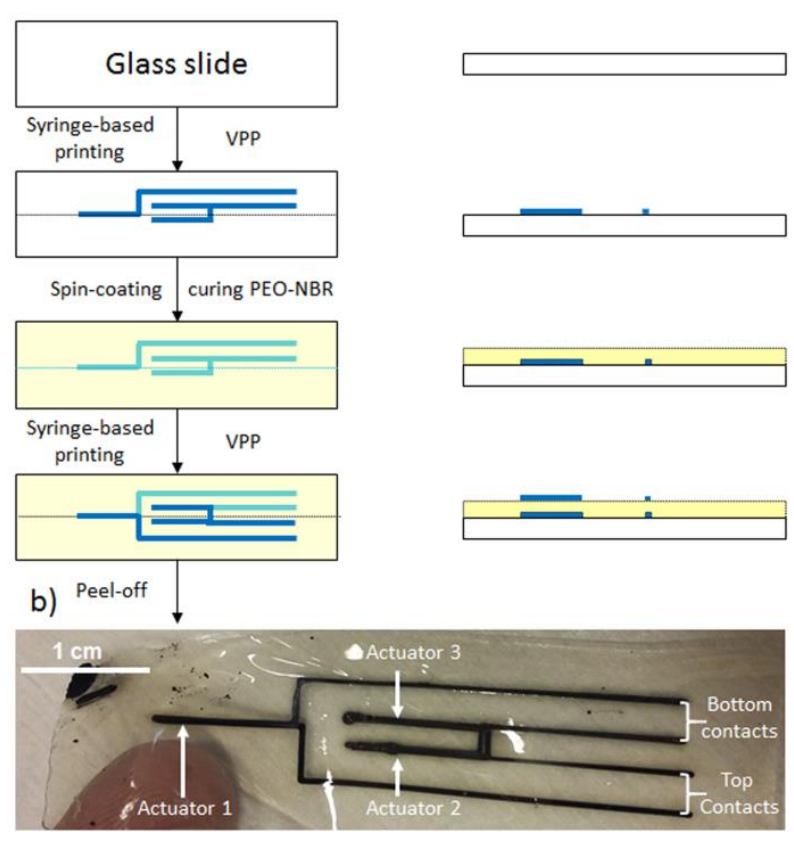

Figure 6. a) A schematic of the patterning process for the fabrication of a CP trilayer actuator device. The bottom electrodes were deposited by syringe-based printing of the oxidant solution on glass, followed by VPP of PEDOT. The second step is the spin coating and the curing of the PEO-NBR membrane. The third step is the patterning of the second layer of electrodes using the same method used for the first electrode layer. Special attention was paid to align the actuator electrodes. The last step is to peel the trilayer membrane from the glass substrate (not shown). b) A photograph showing the finished device, designed as a finger with two joints that can be individually controlled. It comprises 3 actuators, integrated electrical leads, and contacts. Actuator 1 forms the first joint and can be controlled 
via the outermost leads. The second joint is formed by actuators 2 and 3 that are electrically interconnected and can be controlled by the inner leads.

\subsection{Laser ablation}

As the final processing step (after polymerization and before removal from the supporting substrate), the actuator device needs to be cut out from the rest of the membrane to release the actuating beam. Previously, this was done using reactive ion etching (RIE) where the final device structure was defined by a photoresist pattern. ${ }^{26}$ This was possible since the continuous CP layer on top of the SPE protected the SPE from swelling caused by the photoresist processing. However, if the top CP layer, i.e. the layer on top of the SPE, is already patterned, as is the case for our VPP patterned CP electrodes, photoresist cannot be used since parts of the SPE are exposed to the solutions used in the photoresist process, causing swelling. ${ }^{40}$ Here, we used laser ablation to define the final device shape. Laser ablation has a lower resolution than photolithography combined with RIE, but is more versatile in rapid prototyping applications. We have patterned an individual beam with $60 \times 500 \times 2000 \mu \mathrm{m}^{3}$ geometry and a contact pad (Figure $7 b$ ), and millimeter-sized hands (Figure 7d) to illustrate the usefulness of this technique for this type of device. After swelling the devices in the ionic liquid 1-Ethyl-3-methylimidazolium bis(trifluoromethylsulfonyl)imide (EMITFSI), we evaluated the actuation of these hands by connecting them at the palm (Video S1). The actuation properties of this milli/micro device are interesting. For the hand, we achieved $\sim 1 \mathrm{~mm}$ displacement when stimulating with $\mathrm{a} \pm 2 \mathrm{~V}$ square wave potential at $0.1 \mathrm{~Hz}$, which gives a strain of $\varepsilon=2,2 \%$. (See experimental section for the definition of strain $\varepsilon$. The active length was $2.5 \mathrm{~mm}$ and total actuator thickness $70 \mu \mathrm{m}$.) The performance can be further improved by adding an extra electropolymerization step during the process to provide a more electroactive material. ${ }^{35}$ 
a)

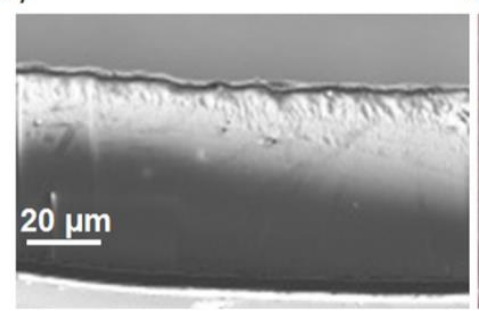

b)

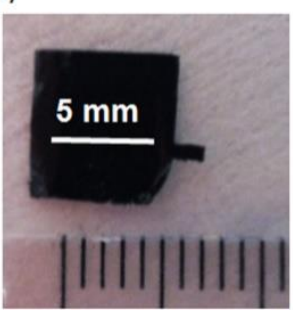

c)

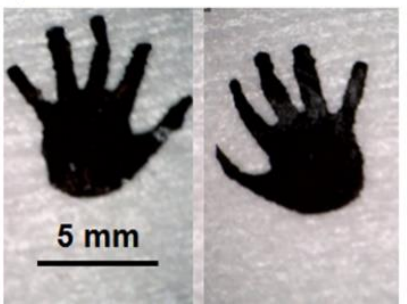

d)

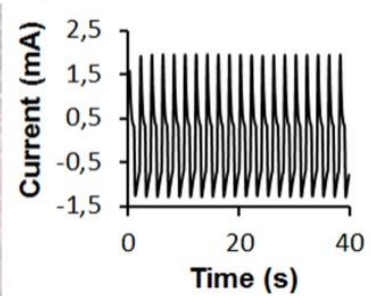

Figure 7. a) Scanning electron microscopy image of the trilayer structure cross-section. b) A $500 \times 2000 \mu \mathrm{m}^{2}$ trilayer bending beam CP actuator of with its contact pad patterned using laser ablation. c) Five-millimeter-wide patterned hands. d) Current signal from the actuation of the hand at a frequency of $1 \mathrm{~Hz}$ and potential amplitude of \pm 2 V. Video S-1 shows the actuation of one hand.

\subsection{Actuation test}

Figure 8a shows an array of free-standing syringe-based printed trilayer actuators (10 $\mathrm{mm}$ long, wide and $70 \mu \mathrm{m}$ thick) following the method illustrated in Figure 6. One of these line actuators was manually cut to investigate the actuation properties (Figure 8b). PPy electrochemical deposition was performed for 2 hours at $0.1 \mathrm{~mA} \mathrm{~cm}^{-2}$ at $-18^{\circ} \mathrm{C}$ from an electrolyte solution (0.1M LiTFSI and $0.1 \mathrm{M}$ pyrrole in propylene carbonate).$^{28}$ The fabricated trilayer was immersed into the ionic liquid EMITFSI until absorption (swelling) ceased ( $\sim 6 \mathrm{hr}$ ) and its actuation behavior was characterized by applying a $\pm 1 \mathrm{~V}$ square wave potential at $0.025 \mathrm{~Hz}$ to the electrodes (Figure $8 \mathrm{~b}$ ). The total thickness of this actuator was $\sim 100 \mu \mathrm{m}$. The large bending typical of $\mathrm{CP}$ actuators was also observed for these printed actuators. A maximum deflection/bending of $10 \mathrm{~mm}(\varepsilon=2 \%)$ was achieved similar to previous unpatterned devices, ${ }^{24}$ indicating that the patterned actuators perform similarly to their unpatterned counterparts. Patterning in this manner eliminates many of the negative effects inherent in other techniques, 
opening a new path for bottom-up fabrication of individually fabricated polymer actuators. To demonstrate the potential of combining the two CP patterning techniques presented with laser ablation, a device containing two individually addressable trilayer actuators has been fabricated (Figure 8c). Two adjacent trilayer actuators from the array of actuators shown in the Figure 8a have been neatly cut from the membrane using laser ablation, leaving a $\sim 9 \mathrm{~mm}$ long segment of the polymer electrolyte layer connected at the bottom, thus forming a device with two individually controlled digits. The device, folded in half so that the actuators oppose one another, clearly demonstrates that with further process and design optimization the combination of the developed CP patterning methods with laser ablation enables the development of complex soft microrobotic devices with individually controlled actuators. 

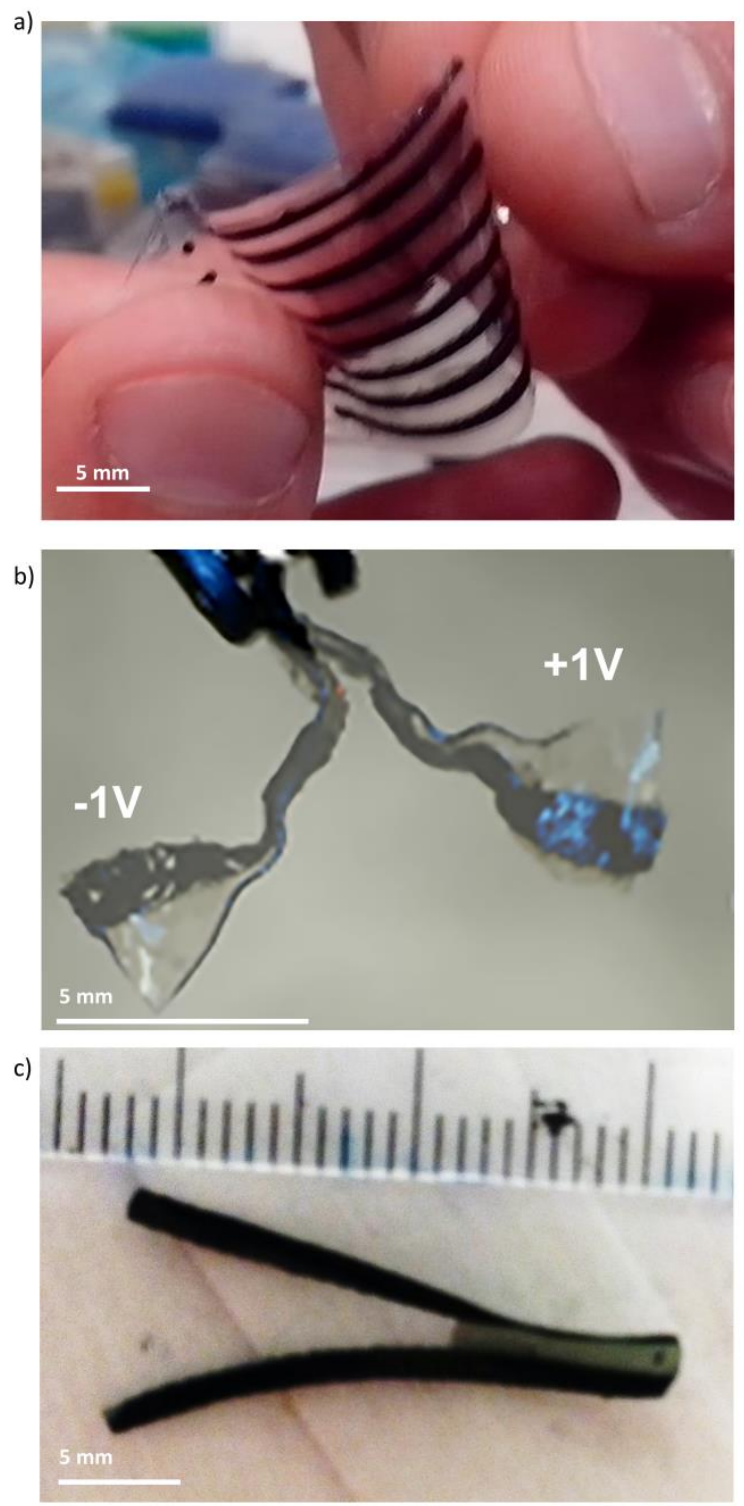

Figure 8. a) An array of syringe printed, individually controllable, flexible actuators. b) Juxtaposition of two photographs showing the extreme states of the actuated trilayer device with an applied potential $\pm 1 \mathrm{~V}$ at $0.025 \mathrm{~Hz}$ (presence of PPy layer). c) Top view of a device with two individually controllable digits. The device consists of two juxtaposed trilayer actuators, connected at the bottom by a $\sim 9 \mathrm{~mm}$ long segment of the polymer electrolyte layer, cut from the array of actuators shown in (a) using laser ablation.

\section{Conclusion}


We have demonstrated two complementary techniques $-\mu$-contact printing and syringe-based printing - to create highly conducting CP patterns via Vapor Phase Polymerization. This was enabled through a study that yielded an understanding of the tunable wetting of the oxidation solution on the various substrates involved and a subsequent optimization of the process parameters, including the PEGM-PEGDM content. This improved VPP technique achieved a more uniform, higher conductivity film, with tuneable wetting for use on various substrates. Patterns down to $100 \mu \mathrm{m}$ can be fabricated with a good resolution using $\mu$-contact printing and VPP. Line widths down to $1 \mathrm{~mm}$ can easily be achieved using syringe-based printing and VPP. $\mu \mathrm{CP}$ combined with VPP is useful for fabricating micro-electrochemical devices of any arbitrary shape with high resolution, and especially allows complete layouts to be printed in a single patterning step. Syringe-based printing is very promising for fabricating functional devices at the millimeter and submillimeter scales, especially for multilayered systems. Moreover, syringebased printing has the potential for use with 3-D printing to obtain 3-D electrodes and even encapsulated devices. In these first demonstrations, the resolution was not optimized and can likely be improved by further adjusting the processing parameters such as the stamps patterns and their surface density, the formulation of the oxidant solution, the choice of the substrate and the chemical functionalization of the surface of the stamp. Finally, we showed that it is possible to fabricate fully functional electrochemical devices, in this case trilayer actuators, using the new printing methods. In combination with laser ablation this should enable the fabrication of complex soft micro-tools. The devices fabricated using these newly developed patterning methods function as good as non-patterned devices, demonstrating that the patterning does not result in any significant negative effects, thus opening the way of new bottom-up fabrication of 
individually fabricated electrochemical devices such as soft actuators, flexible batteries, and stretchable components for bioelectronics and soft robotics.

\section{Experimental}

Materials: The oxidant solution, $40 \mathrm{wt} \% \mathrm{Fe}(\mathrm{Tos})_{3}$ solution in ethanol/Butanol mixture was obtained from Heraeus corporation. Pyrrole was obtained from Sigma-Aldrich, distilled and stored at $-18{ }^{\circ} \mathrm{C}$ prior to use. Azobisisobutyronitrile (AIBN, Sigma-Aldrich) was recrystallized in methanol prior to use. Cyclohexanone (Acros, 99.8\%), poly(ethylene glycol)dimethacrylate. (PEGDM, Mn $=750 \mathrm{~g} \mathrm{~mol}^{-1}$, Aldrich), poly(ethylene glycol) methyl ethermethacrylate (PEGM, $\mathrm{Mn}=475 \mathrm{~g} \mathrm{~mol}^{-1}$ ), polyvinylalcohol $\left(\mathrm{PVA}\right.$, Mowiol 4-88, $\mathrm{Mw} \approx 31000 \mathrm{~g} \cdot \mathrm{mol}^{-1}$ ) were purchased from Sigma-Aldrich. Nitrile butadiene rubber (NBR) with $44 \mathrm{wt} \%$ acrylonitrile content was obtained from Lanxess.

3,4-azobisisobutyronitrile (AIBN, Sigma-Aldrich) was recrystallized in methanol prior to use.

PVA deposition: A 10 wt.\% PVA solution was prepared by dissolving PVA in water while stirring for $24 \mathrm{~h}$ at $95{ }^{\circ} \mathrm{C}$. The PVA layer was deposited on a glass substrate from the PVA solution using a spin coater (Ramp $500 \mathrm{rpm} \cdot \mathrm{s}^{-1}, 500 \mathrm{rpm}$ for $30 \mathrm{~s}$ ). A curing step of 5 minutes at $50{ }^{\circ} \mathrm{C}$ was applied to dry the film.

PEO-NBR semi-IPN synthesis ${ }^{32}$ PEO/NBR solid polymer electrolyte composition: PEO-NBR 50/50 semi-IPN film was obtained by an in-situ polymerization. First the linear NBR solution was prepared by dissolving NBR in cyclohexanone (1:5 weight ratio). After complete dissolution, PEO network precursors (50 wt.\% vs NBR) were introduced by adding PEGM monomer (75 wt.\% of the PEO network), PEGDM crosslinker (25 wt.\% of the PEO network) 
and AIBN (3wt.\% of the methacrylic monomers). The mixture of PEO and NBR was then spincoated. The thickness of the polymer films depends on the selected spin coater speed, time and solution viscosity. The substrate was then kept at $80^{\circ} \mathrm{C}$ for $10 \mathrm{~h}$.

Oxidant solution preparation: The $40 \%$ oxidant solution was poured in a vial. The ethylene oxide acrylate oligomers (PEGM-PEGDM) were then added to the solution and stirred with a magnetic bar. The ethylene oxide acrylate oligomers derivatives had a fixed proportion of monomers PEGM/PEGDM of 75/25 for each of the prepared solutions.

Vapor phase polymerization: The oxidant solution was spincoated on the substrate at $1000 \mathrm{rpm}$ for $30 \mathrm{~s}$ on a POLOS spin-coater on glass slides. The coated substrates were placed on the hot plate in the vacuum oven $(1 \mathrm{mPa})$ containing a Petri dish with 2 drops of EDOT and maintained at $40{ }^{\circ} \mathrm{C}$ for $30 \mathrm{~min}$. Thereafter the substrates were taken out, washed with ethanol and dried at room temperature at $1 \mathrm{mPa}$ for $2 \mathrm{~h}$.

Electropolymerisation: The polypyrrole (PPy) layers were electrodeposited in a two-electrode configuration at a constant current of $0.1 \mathrm{~mA} \cdot \mathrm{cm}^{-2}$ for 2 hours using an Ivium compactstat or IviumStat (Ivium Technologies, Eindhoven, The Netherlands) on the VPP PEDOT layer from a 0.1 M Pyrrole, 0.1 M LiTFSI and $1 \mathrm{wt} \%$ water in a propylene carbonate (PC) solution for 2 hours at $-18{ }^{\circ} \mathrm{C}$. A stainless-steel mesh was used as the counter electrode.

Laser ablation: An adaptive laser ablation procedure was implemented, which was able to accommodate the various device layouts. The ablation platform used in this work was a HL405g, from Full Spectrum Laser LLC, which includes a $40 \mathrm{~W} \mathrm{CO}_{2}$ laser operated in raster mode at 500 ppi and $100 \%$ power. In these conditions, the devices were properly trimmed without 
producing damage by overheating the materials, since in the raster mode the laser only fires for few ms in each location.

The cutting path was adapted to each particular device by first scanning (Konica Minolta C284 PS flatbed scanner) each VPP PEDOT pattern, and then directly drawing the desired laser path on the digitalized image of each geometry using Photoshop. The result of the drawing procedure was then transformed in a bitmap file (.bmp), which can be interpreted by the printer to command the laser path.

Final adjustments, such as printing direction, scaling and thresholding (grey level selected for black \& white transition) were directly performed in the laser control software (Full Spectrum RetinaEngrave3D).

$\mu \mathrm{CP}$ : The PDMS precursors (after mixing and de-gazing) were polymerized on top of the template (silicon wafer with SU-8 pattern). The PDMS stamp was removed from the template and then treated for $30 \mathrm{~s}$ in an $\mathrm{O}_{2}$ plasma.

The different oxidant solutions were spin coated at $2000 \mathrm{rpm} / 500 \mathrm{rpm} \cdot \mathrm{s}^{-1}$ for $30 \mathrm{~s}$ on glass slides. By stamping the PDMS stamp on the iron tosylate spincoated glass slide, the PDMS stamp got inked. The oxidant inked stamp can then pattern the PVDF and PVA substrates using $\mu \mathrm{CP}$. The oxidant pattern underwent VPP in the following step.

Syringe-based printing: Oxidant solutions were deposited using a syringe-based printer (Seraph Robotics Inc. Fab@Home Model 3), using a 200 m-diameter nozzle (straight cannula, blunt end tip, Fisnar Inc.). The different structures were printed using a deposition rate of $3.93 \cdot 10^{-5} \mathrm{~m}^{3} \cdot \mathrm{s}^{-1}$ 
and translation speed of $3 \mathrm{~mm} \cdot \mathrm{s}^{-1}$ at $21^{\circ} \mathrm{C}$. The printed oxidant pattern underwent VPP in the following step.

Contact angle $(\theta)$ measurements were taken with a camera just after the deposition of the droplet on the substrate. Measurements were made on three samples.

Actuation test: Ivium compactstat or IviumStat (Ivium technologies) was used to electrically address the actuators. Gold coated kelvin clips were used to directly contact the actuators. The displacement was recorded with a mobile phone camera.

Strain $\varepsilon$ : The strain is calculated from the following expression for the strain at any location along the actuator length $\mathrm{L}^{41,42}$;

$\varepsilon(\mathrm{x})=2 \frac{h(L-x)^{2}}{L^{4}} y$ tip; $\mathrm{x}=0 ;$ where the variable $\mathrm{x}$ starts from the fixed end of the actuator, $\mathrm{h}$ and ytip are the total thickness of the actuator and tip displacement of the actuator in the transverse direction, respectively.

\section{ASSOCIATED CONTENT}

\section{Supporting information}

Non-essential figures and descriptions that complement the findings and discussion of the manuscript: PEDOT micropatterned surfaces by micro-contact printing on different substrates (PDF); Video of a laser cut actuating hand.

\section{AUTHOR INFORMATION}

\section{Corresponding Author}


*alexandre.khaldi@imt-atlantique.fr, edwin.jager@liu.se

Linköping University, Department of Physics, Chemistry and Biology, 58183 Linköping, Sweden

\section{$\dagger$ Present address}

Alexandre Khaldi : IMT Atlantique Bretagne-Pays de la Loire, Campus de Brest, Technopôle Brest-Iroise, CS 83818, 29238 Brest cedex 03, France

Ali Maziz : LAAS, 7, Avenue du colonel Roche, BP 54200, 31031, Toulouse Cedex 4, France

\section{Author Contributions}

The manuscript was written through contributions of all authors. All authors have given approval to the final version of the manuscript.

\section{Funding Sources}

This study was financially supported by Linköping University, COST Action MP1003 ESNAM (European Scientific Network for Artificial Muscles), the Swedish Research Council (VR 2010-6672, 2014-3079, 2015-03298), the Knut \& Alice Wallenberg Stiftelse (LiU-2010-00318 \& LiU-2012- 01361), and the EU FP7 Marie Curie action IEF (625923 POLYACT).

\section{ACKNOWLEDGMENT}

The authors acknowledge Anthony Turner for his support. 


\section{REFERENCES}

(1) Smela, E. Conjugated Polymer Actuators for Biomedical Applications. Adv. Mater. 2003, $15,481-494$.

(2) Gelmi, A.; Cieslar-pobuda, A.; Muinck, E. De; Los, M.; Rafat, M.; Jager, E. W. H. Direct Mechanical Stimulation of Stem Cells : A Beating Electromechanically Active Scaffold for Cardiac Tissue Engineering. 2016, 1471-1480.

(3) Stavrinidou, E.; Gabrielsson, R.; Gomez, E.; Crispin, X.; Nilsson, O.; Simon, D. T.; Berggren, M. Electronic Plants. Sci. Adv. 2015, 1 (10), e1501136.

(4) Wadhwa, R.; Lagenaur, C. F.; Tracy, X. Electrochemically Controlled Release of Dexamethasone from Conducting Polymer Polypyrrole Coated Electrode. J. Control. Release 2006, 110, 531-541.

(5) Williamson, A.; Rivnay, J.; Kergoat, L.; Jonsson, A.; Inal, S.; Uguz, I.; Ferro, M.; Ivanov, A.; Sjöström, T. A.; Simon, D. T.; et al. Controlling Epileptiform Activity with Organic Electronic Ion Pumps. Adv. Mater. 2015, 27 (20), 3138-3144.

(6) Ouyang, L.; Wei, B.; Kuo, C.; Pathak, S.; Farrell, B.; Martin, D. C. Enhanced PEDOT Adhesion on Solid Substrates with. Sci. Adv. 2017, 3 (March), 1-12.

(7) Hardy, J. G.; Lee, J. Y.; Schmidt, C. E. Biomimetic Conducting Polymer-Based Tissue Scaffolds. Curr. Opin. Biotechnol. 2013, 24 (5), 847-854.

(8) Winther-Jensen, B.; Winther-Jensen, O.; Forsyth, M.; MacFarlane, D. R. High Rates of Oxygen Reduction over a Vapor Phase-Polymerized PEDOT Electrode. Science (80-. ). 
2008, 321 (5889), 671-674.

(9) Fabretto, M. V.; Evans, D. R.; Mueller, M.; Zuber, K.; Hojati-Talemi, P.; Short, R. D.; Wallace, G. G.; Murphy, P. J. Polymeric Material with Metal-Like Conductivity for Next Generation Organic Electronic Devices. Chem. Mater. 2012, 24 (20), 3998-4003.

(10) Maziz, A.; Plesse, C.; Soyer, C.; Cattan, E.; Vidal, F. Top-down Approach for the Direct Synthesis, Patterning, and Operation of Artificial Micromuscles on Flexible Substrates. ACS Appl. Mater. Interfaces 2016, 8 (3), 1559-1564.

(11) Choi, J. S.; Cho, K. Y.; Yim, J.-H. Micro-Patterning of Vapor-Phase Polymerized poly(3,4-Ethylenedioxythiophene) (PEDOT) Using Ink-Jet Printing/soft Lithography. Eur. Polym. J. 2010, 46 (3), 389-396.

(12) Smela, E.; Inganäs, O.; Lundström, I. Controlled Folding of Micrometer-Size Structures. Science 1995, 268, 1735-1738.

(13) Edberg, J.; Iandolo, D.; Brooke, R.; Liu, X.; Musumeci, C.; Andreasen, J. W.; Simon, D. T.; Evans, D.; Engquist, I.; Berggren, M. Patterning and Conductivity Modulation of Conductive Polymers by UV Light Exposure. Adv. Funct. Mater. 2016, 26 (38), 69506960.

(14) Smela, E. Microfabrication of PPy Microactuators and Other Conjugated Polymer Devices. J. Micromechanics Microengineering 1999, 9 (1), 1-18.

(15) Jager, E. W. H. Microrobots for Micrometer-Size Objects in Aqueous Media: Potential Tools for Single-Cell Manipulation. Science (80-. ). 2000, 288 (5475), 2335-2338. 
(16) Jager, E. W. H.; Smela, E.; Inganäs, O. Microfabricating Conjugated Polymer Actuators. Science (80-. ). 2000, 290 (5496), 1540-1545.

(17) Lee, K. K. C.; Herman, P. R.; Shoa, T.; Haque, M.; Madden, J. D. W.; Yang, V. X. D. Microstructuring of Polypyrrole by Maskless Direct Femtosecond Laser Ablation. $A d v$. Mater. 2012, 24 (9), 1243-1246.

(18) Khaldi, A.; Plesse, C.; Soyer, C.; Cattan, E.; Vidal, F.; Legrand, C.; Teyssié, D. Conducting Interpenetrating Polymer Network Sized to Fabricate Microactuators. Appl. Phys. Lett. 2011, 98 (16), 164101.

(19) Tehrani, P.; Robinson, N. D.; Kugler, T.; Remonen, T.; Hennerdal, L.; Häll, J.; Malmström, A.; Leenders, L.; Berggren, M. Patterning Polythiophene Films Using Electrochemical over-Oxidation. Smart Mater. Struct. 2005, 14 (4), N21.

(20) Berg, H.; Schubert, M.; Friedrich, S.; Bock, K. Screen Printed Conductive Pastes for Biomedical Electronics. In 39th International Spring Seminar on electronics Technology (ISSE), IEEE; 2016; pp 1-6.

(21) Palumbiny, C. M.; Heller, C.; Scha, C. J.; Ko, V.; Santoro, G.; Roth, S. V; Mu, P. Molecular Reorientation and Structural Changes in Cosolvent- Treated Highly Conductive PEDOT: PSS Electrodes for Flexible Indium Tin Oxide-Free Organic Electronics. $J$. Phys. Chem. C 2014, 118, 13598-13606.

(22) Palumbiny, C. M.; Liu, F.; Russell, T. P.; Hexemer, A.; Wang, C.; Müller-buschbaum, P. The Crystallization of PEDOT : PSS Polymeric Electrodes Probed In Situ during Printing COMMUNICATION. Adv. Mater. 2015, 27, 3391-3397. 
(23) Maziz, A.; Plesse, C.; Soyer, C.; Chevrot, C.; Teyssié, D.; Cattan, E.; Vidal, F. Demonstrating $\mathrm{kHz}$ Frequency Actuation for Conducting Polymer Microactuators. $A d v$. Funct. Mater. 2014, 24 (30), 4851-4859.

(24) Vidal, F.; Plesse, C.; Teyssié, D.; Chevrot, C. Long-Life Air Working Conducting SemiIPN/ionic Liquid Based Actuator. Synth. Met. 2004, 142, 287-291.

(25) Vidal, F.; Aubert, P.; Beouch, L.; Palaprat, G.; Verge, P.; Yammine, P.; Citerin, J.; Kheddar, A.; Sauques, L. Semi-Interpenetrating Polymer Networks : A Versatile Concept for the Design of Optical or Mechanical Electroactive Devices Claude Chevrot a and Dominique Teyssi E. 2010, No. February, 313-320.

(26) Khaldi, A.; Maziz, A.; Plesse, C.; Soyer, C.; Vidal, F.; Cattan, E. Synergetic PEDOT Degradation during a Reactive Ion Etching Process. Sensors Actuators B Chem. 2016, $229,635-645$.

(27) Jager, E. W. H.; Masurkar, N.; Nworah, N. F.; Gaihre, B.; Alici, G.; Spinks, G. M. Patterning and Electrical Interfacing of Individually Controllable Conducting Polymer Microactuators. Sensors Actuators B Chem. 2013, 183, 283-289.

(28) Khaldi, A.; Maziz, A.; Alici, G.; Spinks, G. M.; Jager, E. W. H. Bottom-up Microfabrication Process for Individually Controlled Conjugated Polymer Actuators. Sensors Actuators B Chem. 2016, 230, 818-824.

(29) Khaldi, A.; Maziz, A.; Alici, G.; Spinks, G. M.; Jager, E. W. H. Soft, Flexible Micromanipulators Comprising Polypyrrole Trilayer Microactuators. In SPIE Smart Structures and Materials + Nondestructive Evaluation and Health Monitoring; Bar-Cohen, 
Y., Ed.; International Society for Optics and Photonics, 2015; p 94301R.

(30) Winther-Jensen, B.; West, K. Vapor-Phase Polymerization of 3,4Ethylenedioxythiophene: A Route to Highly Conducting Polymer Surface Layers. Macromolecules 2004, 37, 4538-4543.

(31) Goujon, L. J.; Khaldi, A.; Maziz, A.; Nguyen, G. T. M.; Aubert, P.; Chevrot, C.; Teyssié, D. Flexible Solid Polymer Electrolytes Based on Nitrile Butadiene Rubber / Poly(ethyleneoxide) Interpenetrating Polymer Networks Containing Either LiTFSI or EMITFSI. Macromolecules 2011, 44, 9683-9691.

(32) Festin, N.; Maziz, A.; Plesse, C.; Teyssié, D.; Chevrot, C.; Vidal, F. Robust Solid Polymer Electrolyte for Conducting IPN Actuators. Smart Mater. Struct. 2013, 22 (10), 104005.

(33) Gaihre, B.; Alici, G.; Spinks, G. M.; Cairney, J. M. Pushing the Limits for Microactuators Based on Electroactive Polymers. J. Microelectromechanical Syst. 2012, 21 (3), 574-585.

(34) Fukushima, T.; Asaka, K.; Kosaka, A.; Aida, T. Fully Plastic Actuator through Layer-byLayer Casting with Ionic-Liquid-Based Bucky Gel. Angew. Chemie Int. Ed. 2005, 44 (16), $2410-2413$.

(35) Khaldi, A.; Falk, D.; Maziz, A.; Jager, E. W. H. Fabrication and Adhesion of Conjugated Polymer Trilayer Structures for Soft, Flexible Micromanipulators. In SPIE Smart Structures and Materials + Nondestructive Evaluation and Health Monitoring; Bar-Cohen, Y., Vidal, F., Eds.; International Society for Optics and Photonics, 2016; p 97980N.

(36) Bubnova, O.; Khan, Z. U.; Wang, H.; Braun, S.; Evans, D. R.; Fabretto, M.; Hojati- 
Talemi, P.; Dagnelund, D.; Arlin, J.-B.; Geerts, Y. H.; et al. Semi-Metallic Polymers. Nat. Mater. 2013, 13 (2), 190-194.

(37) Ugur, A.; Katmis, F.; Li, M.; Wu, L.; Zhu, Y.; Varanasi, K. K.; Gleason, K. K. LowDimensional Conduction Mechanisms in Highly Conductive and Transparent Conjugated Polymers. Adv. Mater. 2015, 27 (31), 4604-4610.

(38) Lee, S.; Paine, D. C.; Gleason, K. K. Heavily Doped poly(3,4-Ethylenedioxythiophene) Thin Films with High Carrier Mobility Deposited Using Oxidative CVD: Conductivity Stability and Carrier Transport. Adv. Funct. Mater. 2014, 24 (45), 7187-7196.

(39) Temmer, R.; Maziz, A.; Plesse, C.; Aabloo, A.; Vidal, F.; Tamm, T. In Search of Better Electroactive Polymer Actuator Materials: PPy versus PEDOT versus PEDOT-PPy Composites. Smart Mater. Struct. 2013, 22 (10), 104006.

(40) Jager, E. W. H.; Maziz, A.; Khaldi, A. Conducting Polymers as EAPs: Microfabrication. In Electromechanically Active Polymers; Springer International Publishing: Cham, 2016; pp 293-318.

(41) Alici, G. An Effective Modelling Approach to Estimate Nonlinear Bending Behaviour of Cantilever Type Conducting Polymer Actuators. Sens. Actuators B Chem. 2009, 141, 284292.

(42) Gaihre, B.; Alici, G.; Spinks, G. M.; Cairney, J. M. Synthesis and Performance Evaluation of Thin Film PPy-PVDF Multilayer Electroactive Polymer Actuators. Sens. Actuators A, 2011, 165, 321-328. 
TOC:

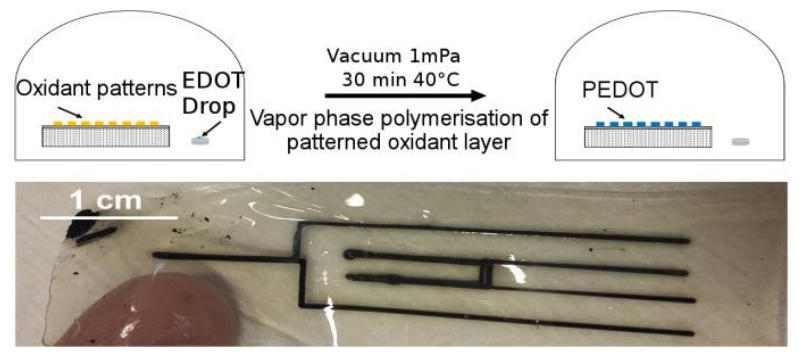

